\title{
An Improved Traveling-Wave-Based Fault Location Method with Compensating the Dispersion Effect of Traveling Wave in Wavelet Domain
}

\author{
Huibin Jia \\ School of Electrical \& Electronic Engineering, North China Electric Power University, Baoding 071003, China \\ Correspondence should be addressed to Huibin Jia; huibin.jia@foxmail.com
}

Received 23 July 2016; Revised 24 December 2016; Accepted 16 January 2017; Published 8 February 2017

Academic Editor: Ruben Specogna

Copyright (C) 2017 Huibin Jia. This is an open access article distributed under the Creative Commons Attribution License, which permits unrestricted use, distribution, and reproduction in any medium, provided the original work is properly cited.

\begin{abstract}
The fault generated transient traveling waves are wide band signals which cover the whole frequency range. When the frequency characteristic of line parameters is considered, different frequency components of traveling wave will have different attenuation values and wave velocities, which is defined as the dispersion effect of traveling wave. Because of the dispersion effect, the rise or fall time of the wavefront becomes longer, which decreases the singularity of traveling wave and makes it difficult to determine the arrival time and velocity of traveling wave. Furthermore, the dispersion effect seriously affects the accuracy and reliability of fault location. In this paper, a novel double-ended fault location method has been proposed with compensating the dispersion effect of traveling wave in wavelet domain. From the propagation theory of traveling wave, a correction function is established within a certain limit band to compensate the dispersion effect of traveling wave. Based on the determined arrival time and velocity of traveling wave, the fault distance can be calculated precisely by utilizing the proposed method. The simulation experiments have been carried out in ATP/EMTP software, and simulation results demonstrate that, compared with the traditional traveling-wave fault location methods, the proposed method can significantly improve the accuracy of fault location. Moreover, the proposed method is insensitive to different fault conditions, and it is adaptive to both transposed and untransposed transmission lines well.
\end{abstract}

\section{Introduction}

Power systems have grown rapidly over the last few decades, and the number and length of transmission lines increased. Transmission lines are exposed in the field, and, especially in the mountains and hilly terrains, they are prone to failure. In this scenario, a fast and accurate fault location technique is essential to reduce the restoration time of power systems, which is important with respect to technical and economic issues. Therefore, the study and development of fault location have been motivated since the 1950s [1]. The traveling-wavebased fault location method has been proposed by lots of researchers because these are insensitive to load flow, transition resistance, wiring ways, and series compensation.

The traveling-wave-based fault location methods for transmission lines can generally be classified as single- and doubleended methods in terms of their different ways of obtaining the fault information. For many years, the single-ended traveling-wave-based methods were recognized by utilities as a good way to overcome the drawbacks of impedance-based approaches [2]. However, these methods commonly have problems of distinguishing between traveling waves reflected from the fault point and from power system terminals, which decreases the reliability of the fault location method [3]. Therefore, double-ended traveling-wave-based methods have been reported for overcoming these drawbacks. The double-ended method employs the data from the two ends of transmission lines; these data are synchronized by using GPS [4]. And the double-ended method has higher reliability and accuracy than the single-ended one.

For the traveling-wave-based fault location method, the accuracy of fault location lies in the arrival time and velocity of traveling wave. Several methods have been proposed to determine the arrival time of traveling wave [5-10]. Wavelet transform has strong time-frequency analysis capability, which can effectively improve the accuracy of the 
singular value detection. Therefore, it has been firstly used for determining the arrival time of traveling wave, which is represented by the wavelet modulus maxima point [5] Hence, several researchers have exploited the continuous wavelet transform to extract the arrival time of traveling wave [6-10]. As a matter of fact, it can be easier to extract the singular value point when mother wavelet is similar to traveling wave. Therefore, the mother wavelet has been extracted from the fault transient signal to improve the accuracy of fault location in distribution network [11, 12]. All these methods described above determine the arrival time of wavefront with singular value detection algorithm without considering different frequency components of traveling wave having different arrival times.

On the other hand, the wave velocity also directly affects the accuracy of fault location. Some researchers have proposed some methods which are insensitive to the velocity of traveling wave [13-15]. The method proposed in [13] has exploited the three-terminal synchronized transient data, which increases the cost of fault location and decreases the reliability of fault location. The method proposed in [14] is excessively dependent on the first consecutive transient wavefronts of traveling wave, which makes it difficult to distinguish the wavefront of the reflected traveling wave. The method in [15] needs a dedicated communication system to locate the faults, but unfortunately the latency of the dedicated communication system is uncertain, which increases the error of fault location. Currently, the light speed is selected in most traveling-wave-based fault location methods. A few researchers have made significant attempts to determine the wave velocity. Reference [16] calculated the wave velocity with the arrival time of initial wave at nonfault phase when an out-area fault happens. Reference [17] calculated the wave velocity with high-frequency signal generated by autoreclosure. There is a certain difference between the fault signal and the analyzed high-frequency signal. Lots of experts have made discussions and attempts on the problem of determining the wave velocity, but there is still not a brilliant solution. Moreover, these methods have not also considered the different frequency components having different propagation velocities.

When a fault happens, the generated traveling wave contains a lot of frequency components, which can be viewed as very important fault information. Because of the frequencydependent parameters of transmission line, each component has different velocity and attenuation. This phenomenon is defined as traveling-wave dispersion effect [18-20], which distorts the wavefront of traveling wave and lengthens the fall or rise time of the wavefront. Therefore, it is difficult to accurately determine the arrival time and velocity of traveling wave. In [18-20], the authors just analyzed the influence of the dispersion effect on the traveling-wave-based fault location and did not provide the perfect solution. In this paper, a correction method for dispersion effect of traveling wave is proposed. The correction method can shorten the fall or rise time of the wavefront and enhance the singularity of traveling wave. Thus, the accuracy and reliability of fault location can be improved significantly.

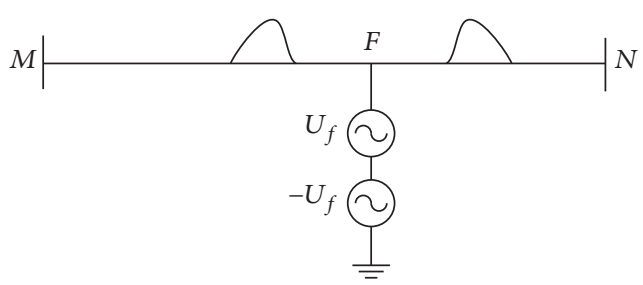

Figure 1: Traveling-wave theory.

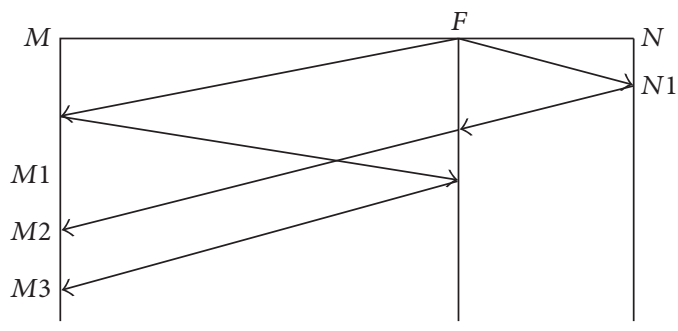

FIGURE 2: Refection and refraction of traveling wave.

The paper is organized as follows. Section 2 introduces the principle of traveling-wave-based fault location. Section 3 analyzes dispersion effect of traveling wave. Section 4 proposes a correction method for dispersion effect of traveling wave in detail. Section 5 shows the analysis of simulations and results. Finally, Section 6 is the conclusion.

\section{Principle of Traveling-Wave-Based Fault Location}

2.1. Traveling-Wave Theory. When a fault happens on a transmission line, the generated voltage and current surges will travel towards both ends of the line. This is equivalent to generating a virtual voltage source at the fault point, as shown in Figure 1. The traveling wave is driven by the voltage source and travels towards both ends of the line. Its velocity is close to the velocity of light.

Refraction and reflection will occur at the fault point, ends of lines, and other discontinuity points. Thus, the generated reflected and refracted wave will propagate along the transmission line, as shown in Figure 2. The wavefront is detected when the initial traveling wave first reaches $M$-side and $N$-side, denoted as $M 1$ and N1. Then, the signal moves to the fault point from $M$-side ( $N$-side) of the line and returns to the fault point $F$. The refracted wavefront $N 1$ will reach $M$-side, marked as $M 2$. And the reflected wavefront $M 1$ will go back to $M$-side again, marked as $M 3 . M 1$ and $N 1$ are used for double-ended fault location in this paper.

2.2. Double-Ended Traveling-Wave-Based Method. In the double-ended traveling-wave-based method, the distance between a fault point and measuring point at $M$ and $N$ terminals can be obtained according to

$$
\begin{aligned}
& l_{M}=\frac{1}{2}\left[v\left(t_{M}-t_{N}\right)+L\right] \\
& l_{N}=\frac{1}{2}\left[v\left(t_{N}-t_{M}\right)+L\right],
\end{aligned}
$$


where $l_{M}$ and $l_{N}$ are the distance away from the measuring points at $M$ and $N$ terminals to the fault point, respectively. $L$ is the total length of the transmission line. $v$ is the propagation velocity of traveling wave.

2.3. Phase-to-Modal Transformation. There is an electromagnetic coupling effect between transmission lines. Therefore, by using a modal transformation matrix, we can decompose the traveling wave between phases into several independent modes. In the case of transposed transmission lines, the parameters of transmission line are balanced and the modal transformation can be defined as constant and real [21], such as Karrenbauer transformation and Clarke transformation. In this paper, Karrenbauer transformation is adopted for decoupling the transposed transmission lines. The Karrenbauer transformation is as follows:

$$
\left[\begin{array}{c}
U_{0} \\
U_{\alpha} \\
U_{\beta}
\end{array}\right]=\frac{1}{3}\left[\begin{array}{ccc}
1 & 1 & 1 \\
-1 & 0 & 1 \\
0 & -1 & 1
\end{array}\right]\left[\begin{array}{l}
U_{A} \\
U_{B} \\
U_{C}
\end{array}\right],
$$

where $U_{A}, U_{B}$, and $U_{C}$ are the phase voltages, respectively. $U_{0}$ is the ground mode component, and $U_{\alpha}$ and $U_{\beta}$ are two independent mode components, respectively. Each independent mode component has different velocity and attenuation.

For untransposed transmission line, the phase-to-modal transformation matrix is frequency-dependent and unsymmetrical [22]. Fortunately, it is shown that the frequency effect on the modal transformation matrix can be neglected when the frequency is high enough [23]. Therefore, transformation matrix can be approximated as a real and frequencyindependent one. In the case of untransposed transmission line, the Karrenbauer transformation is also adopted in this paper.

2.4. Wavelet Transform. Wavelet transform (WT) is a common mathematical tool for digital signal processing. WT has been widely applied in lots of fields, such as time series analysis, speech processing, digital image processing, and power system transient analysis [24]. The continuous wavelet transform (CWT) of a signal $f(t)$ is the integral of the product between $f(t)$ and the daughter wavelet. The daughter wavelet is constructed by a mother wavelet, which is dilated with a scale parameter $a$ and translated by $b$. Therefore, the mother wavelet of CWT can be defined as (5). Hence,

$$
\begin{aligned}
\operatorname{CWT}(f(t) ; a, b) & =\int_{-\infty}^{\infty} f(t) \Psi_{a, b}^{*}(t) d t \\
\Psi_{a, b}^{*}(t) & =\frac{1}{\sqrt{a}} \Psi\left(\frac{t-b}{a}\right),
\end{aligned}
$$

where $\Psi_{a, b}(t)$ is the mother wavelet and $*$ is the complex conjugate operation. $a$ is a scale factor and $b$ is the transition factor. Wavelet transform has a time-frequency resolution. A short window is used for high frequencies while a long window is for low frequencies. Sharp signal transitions create large-amplitude wavelet coefficients. Large wavelet coefficients can detect and measure short high-frequency variations because they have narrow time localization at high frequency. Therefore, WT is very attractive for the analysis of transient signals [25].

Wavelet transform is regarded as a filter. The filter of wavelet transform can be employed as follows:

$$
\begin{aligned}
g(t) & =\int_{-\infty}^{\infty} f(\tau) h(t-\tau) d \tau \\
G(\omega) & =F(\omega) \cdot H(\omega),
\end{aligned}
$$

where $h(t)$ is the pulse response of the filter and $H(\omega)$ is the frequency response of the filter. Comparing (4) and (6), $h(t)$ can be described as

$$
h(t)=|a|^{-1 / 2} \overline{\psi\left(\frac{-t}{a}\right)} .
$$

$H(\omega)$ is also represented as

$$
H(\omega)=\sqrt{a} \cdot \overline{\Psi(a \omega)}
$$

From the analysis above, wavelet transform can be calculated by using Fast Fourier Transform (FFT) and Inverse Fast Fourier Transform (IFFT). Therefore, wavelet transform of traveling wave can be employed by using the following equation:

$$
\mathrm{CWT}_{a}=\operatorname{IFFT}(f(\omega) H(\omega)),
$$

where $f(\omega)$ is the calculation result of FFT for traveling wave and $\mathrm{CWT}_{a}$ is the result of wavelet transform under the scale $a$ and it is used for fault location. In this paper, the continuous wavelet transform is utilized to extract the wavefront of traveling wave and determine the arrival time of traveling wave.

\section{Analysis of Traveling-Wave Dispersion Effect}

3.1. Analysis of Dispersion Effect in Frequency Domain. The traveling wave has a lot of frequency components. As a matter of fact, the distributed parameters of transmission line are frequency-dependent, which results in different frequency components having different velocities and attenuation values. This phenomenon is defined as the dispersion effect of traveling wave [18].

There are two important parameters for the travelingwave dispersion effect. One is the propagation coefficient, and the other is phase velocity. The propagation coefficient of the $m$-modal component is calculated as follows:

$$
\gamma_{m}(\omega)=\sqrt{\left(R_{m}+j \omega L_{m}\right)\left(G_{m}+j \omega C_{m}\right)},
$$

where $R_{m}, L_{m}, G_{m}$, and $C_{m}$ are the $m$-modal resistance, inductance, conductance, and capacitance, respectively, which are frequency-dependent because of the influence of skin effect [26]. These distributed parameters can be calculated by using Carson formulation according to geometrical parameters of transmission line. It can be observed from 


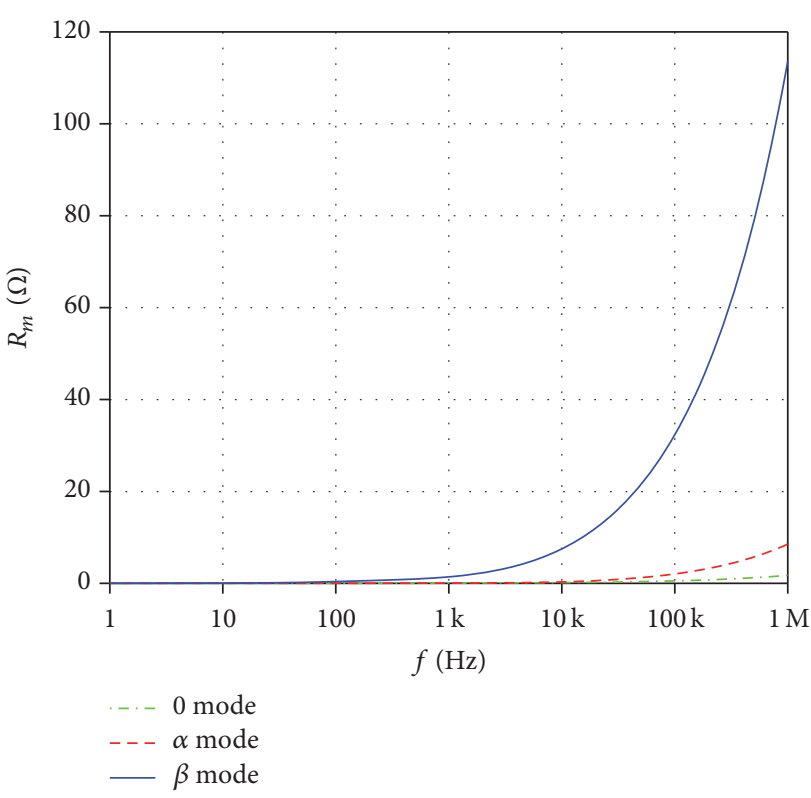

(a) Resistance varied with frequency

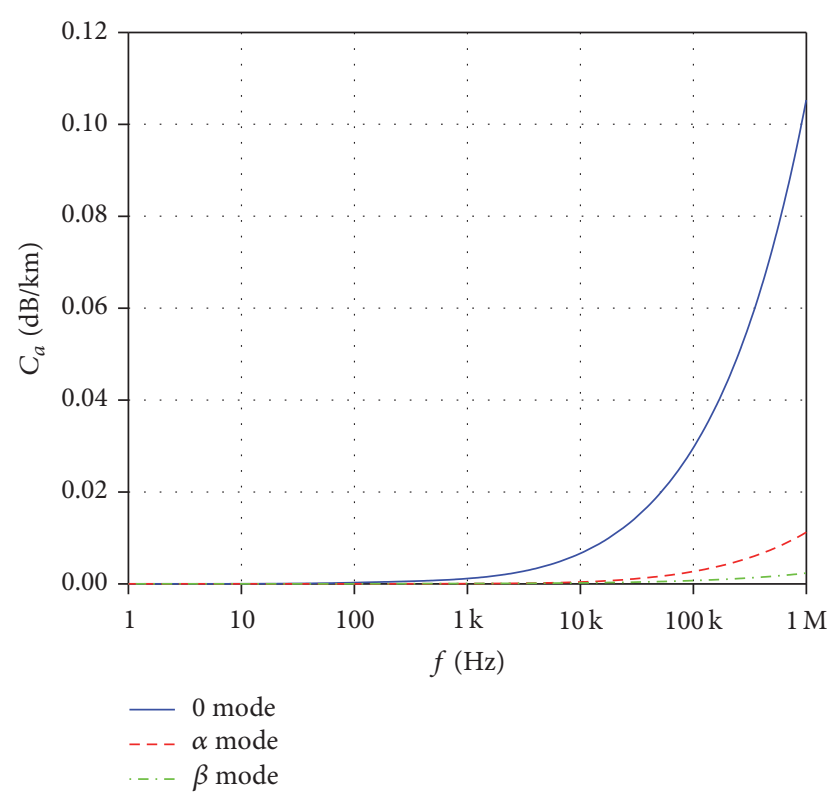

(c) Attenuation coefficient varied with frequency

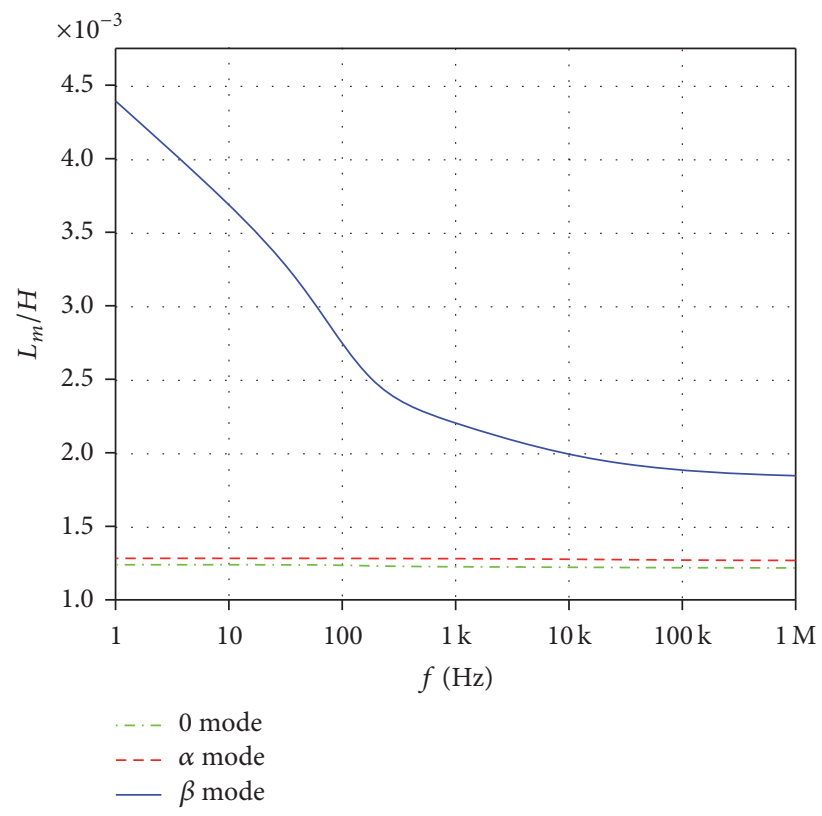

(b) Reactance varied with frequency

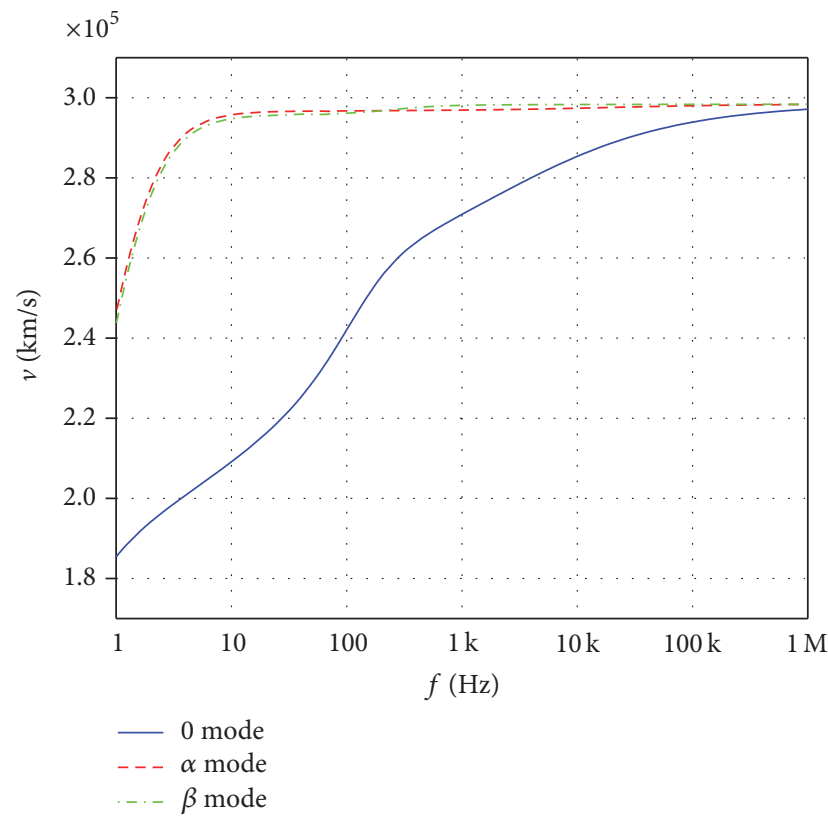

(d) Propagation velocity varied with frequency

FIGURE 3: Frequency-dependent parameters of transmission line.

Figure 3 that the modal resistance increases with the increase of the frequency, and the modal inductance decreases with the increase of frequency.

The propagation coefficient is also described as follows:

$$
\gamma_{m}(\omega)=\alpha_{m}(\omega)+j \beta_{m}(\omega),
$$

where $\alpha_{m}$ is the attenuation coefficient and $\beta_{m}$ is the phase coefficient. They are also frequency-dependent parameters.
The phase velocity can be calculated by phase coefficient $\beta_{m}$, which is defined as

$$
V_{p m}=\frac{\omega}{\beta_{m}} .
$$

The modal attenuation coefficient and phase velocity, which vary with frequencies, are shown in Figures 3(c) and $3(\mathrm{~d})$. The frequency ranges from $1 \mathrm{~Hz}$ to $1 \mathrm{MHz}$.

It can be seen from Figure 3 that the ground-modal parameters are more affected by the frequency than the 
line-modal parameters. The line-modal component is widely used for the traveling-wave-based fault location. Nevertheless, the characteristics of frequency-dependent parameters should not be ignored for improving the accuracy of fault location. For line-modal parameters, attenuation coefficients and propagation velocities increase along with the increase of frequency. For attenuation coefficients, the change of the low frequency is less than that of high frequency; for propagation velocities, the change of the low frequency is greater than that of the high frequency. The characteristic of frequency-dependent parameters makes the rise or fall time of wavefront spread out, which decreases the singularity of transient traveling wave.

3.2. Analysis of Dispersion Effect in Time Domain. In frequency domain, attenuation and wave velocity of traveling wave increase along with frequency. In time domain, the arrival times of different frequency components are not the same. The high-frequency component will arrive at the measuring point first of all, and the low-frequency component will be delayed much more in reaching the measuring point than the high-frequency component. Therefore, all of the detected wavefronts at different measuring points are not ideal step signals; these rise times or fall times get long, which can been seen from Figure 4. In the figure, the signal at the fault point is an ideal step one. At the measuring point 150 kilometers away from the fault point, the fall time of transient traveling wave becomes larger than that at the fault point. At the measuring point 300 kilometers away from the fault point, the fall time of the transient traveling wave becomes larger than that at 150 kilometers. The fall time will become larger and larger with the increase of the distance between the measuring point and the fault point. Thus, the singularity of transient traveling wave gradually decreases. In summary, as a result of the dispersion effect of traveling wave, the wavefront is distorted, which makes it more difficult to be detected. Furthermore, the dispersion effect of traveling wave will decrease the accuracy and reliability of fault location.

\section{Correction Method of Traveling-Wave Dispersion Effect}

4.1. Correction Method of Traveling-Wave Dispersion Effect. The propagation theory of forward and reverse traveling wave is shown in Figure 5. The direction of the "forward" and "reverse" is relative. The component that leaves the terminal is defined as "forward"; the component that enters the terminal from the line is defined as "reverse." The length of transmission line between $M$ and $N$ terminal is $l$.

According to the propagation equation of single conductor line, the voltages $U_{M}$ and $U_{N}$ at $M$ and $N$ terminal can be obtained as follows:

$$
\begin{aligned}
& U_{N}=F_{N}+B_{N}=F_{N}+F_{N} e^{-\gamma(\omega) l} \\
& U_{M}=F_{M}+B_{M}=F_{M}+F_{N} e^{-\gamma(\omega) l},
\end{aligned}
$$

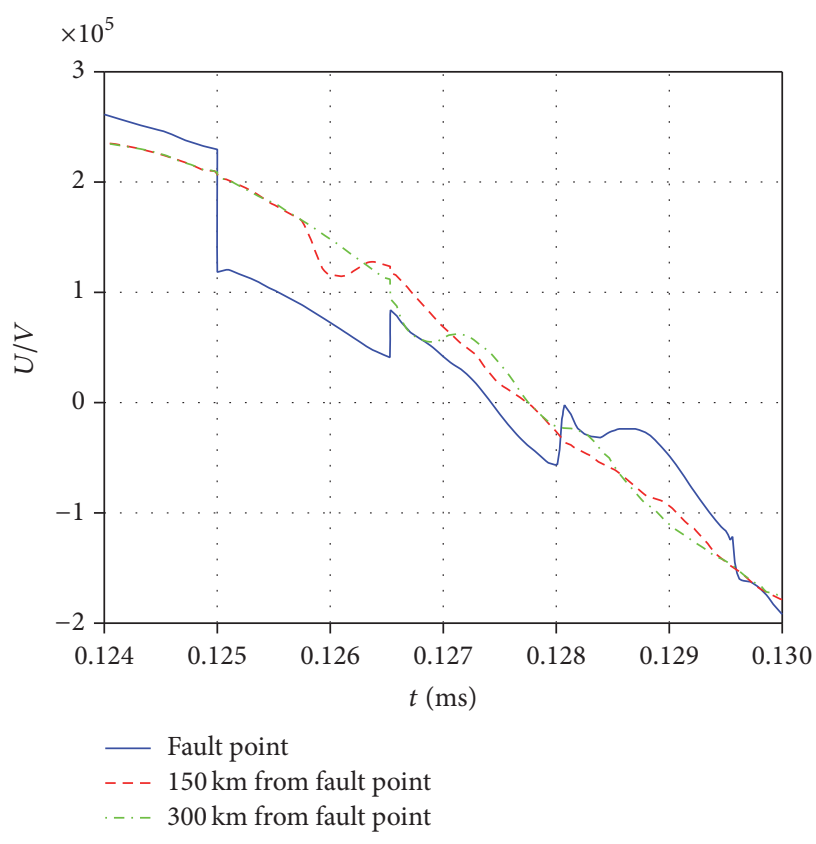

FIGURE 4: $\alpha$-mode wavefronts at different measuring points.

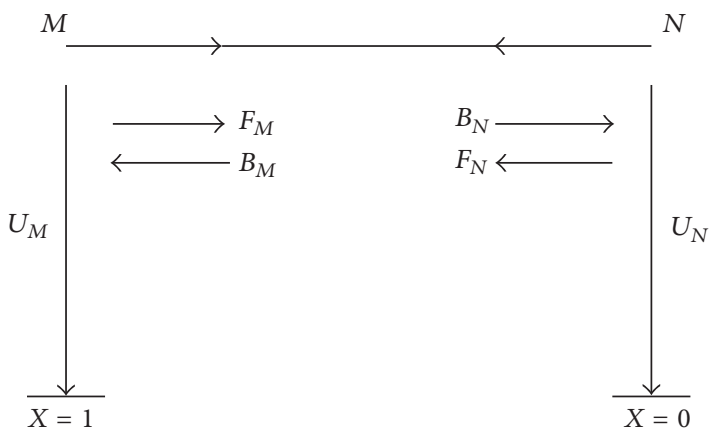

FIGURE 5: The propagation theory of forward and reverse traveling wave.

where $e^{-\gamma(\omega) l}$ is the propagation function. Compared with (14), each reverse traveling wave can be obtained as follows:

$$
\begin{aligned}
& B_{M}=F_{N} e^{-\gamma(\omega) l} \\
& B_{N}=F_{M} e^{-\gamma(\omega) l} .
\end{aligned}
$$

From signal and system theory, the reverse traveling wave $B_{M}$ is the frequency response of the forward traveling wave $F_{N}$; similarly, the reverse traveling wave $B_{M}$ is the frequency response of the forward traveling wave $F_{M}$. For lossy transmission line, $\gamma(\omega)$ varies with frequency. It distorts the wavefront of traveling wave and decreases its singularity. Obviously, $\gamma(\omega)$ causes the dispersion effect of traveling wave.

Likewise, the initial surge of traveling wave, which is used for double-ended fault location method, is the frequency response of the signal at the fault point. Suppose that the distance between the fault point and the measuring point is $L$ and the transient traveling wave at the fault point is referred to as $f(\omega)$ in frequency domain, which is an ideal step signal 
in time domain as shown in Figure 4. Thus, the traveling wave at the measuring points can be described as $f(\omega) e^{-\gamma(\omega) L}$ in frequency domain from the analysis above, and this wave is distorted by $e^{-\gamma(\omega) L}$. The traveling wave at the measuring points can be multiplied by $A(\omega)$, and the traveling wave at the fault point with the constant delay can be obtained, and it is described as follows:

$$
\begin{aligned}
f(\omega) e^{-\gamma(\omega) L} A(\omega) & =f(\omega) e^{-j \omega \tau} \\
\tau & =\frac{L}{v},
\end{aligned}
$$

where $v$ is set as the velocity at $50 \mathrm{~Hz} . f(\omega) e^{-j \omega \tau}$ is the perfect solution for the traveling-wave-based fault location, because it is an ideal step signal and it has perfect singularity and constant delay. In theory, $f(\omega) e^{-j \omega \tau}$ is very suitable for fault location. Consequently, the correction function in frequency domain is obtained as follows:

$$
A(\omega)=Q^{-1}(\omega)=e^{\gamma(\omega) L-j \omega \tau} .
$$

For fault location, $A(\omega)$ multiplies the receiving traveling wave at the measuring point in frequency domain; the perfect signal $f(\omega) e^{-j \omega \tau}$ can be obtained and it is used for the traveling-wave-based fault location. That is to say, all of the frequency components have the same velocity and attenuation to the frequency component at $50 \mathrm{~Hz}$ by using the correction function. When the fault happens on the transmission line, the forward or reverse traveling wave travels towards the measuring points. The distorted wavefront of the initial traveling wave is corrected by the correction function. All frequency components of the initial traveling wave will be postponed for some constant time to reach the measuring point and all of them have constant attenuation values, which make it easy to accurately determine the arrival time and wave velocity. By the correction method proposed in this paper, the singularity of the receiving transient traveling wave is significantly enhanced.

4.2. Implementation of the Correction Method. When a fault happens, the three-phase voltage at $M$ and $N$ measuring terminals can be obtained. Firstly, approximate fault distance can be obtained by using wavelet transform, which is described in Sections 2.2 and 2.4. The approximate fault distance is used for calculating the correction function; secondly, we select several discrete frequencies and calculate the propagation function of each frequency with $R, L$, and $C$ parameters by using Carson formulation, and then the correction function $A(\omega)$ can be obtained by using (18); thirdly, the corrected traveling wave can be obtained according to (16) in frequency domain and the fault location procedure is implemented by wavelet transform again. The specific steps are shown as follows.

Step 1. The three-phase voltage is obtained when a fault happens.

Step 2. The transient three-phase voltages are first decoupled into their independent modal components by using (3), and then $\alpha$-mode traveling wave, $u_{M}(t)$ and $u_{N}(t)$, is used for fault location.

Step 3. $u_{M}(\omega)$ and $u_{N}(\omega)$ of $\alpha$-mode traveling wave can be obtained by Fast Fourier Transform (FFT).

Step 4. At the scale $a$, the frequency response of the mother wavelet function, $H(\omega)$, can be calculated by using (9).

Step 5. At the scale $a$, the decomposition result of wavelet transform can be obtained by IFFT according to (10); this result can be described as follows:

$$
\begin{aligned}
& \mathrm{WT}_{a} u_{M}(t)=\operatorname{IFFT}\left(u_{M}(\omega) \cdot H(\omega)\right) \\
& \mathrm{WT}_{a} u_{N}(t)=\operatorname{IFFT}\left(u_{N}(\omega) \cdot H(\omega)\right) .
\end{aligned}
$$

Step 6. Based on the double-ended fault location theory described in Section 2.2, approximate fault distance, $l_{M}$ and $l_{N}$, can be obtained.

Step 7. Calculate the propagation function based on Carson formulation, and then calculate the corresponding corrected function $A_{M}(\omega)$ and $A_{N}(\omega)$ for $u_{M}(\omega)$ and $u_{N}(\omega)$, respectively.

Step 8. At the scale $a$ of wavelet transform, the corrected decomposition result of wavelet transform can be obtained by IFFT; this result can be shown as follows:

$$
\begin{aligned}
& \mathrm{WT} u_{M}^{\prime}(t)=\operatorname{IFFT}\left(u_{M}(\omega) \cdot A_{M}(\omega) \cdot H(\omega)\right) \\
& \operatorname{WT} u_{N}(t)=\operatorname{IFFT}\left(u_{N}(\omega) \cdot A_{N}(\omega) \cdot H(\omega)\right) .
\end{aligned}
$$

Step 9. Based on the double-ended fault location theory, accurate fault distance, $l_{M}^{\prime}$ and $l_{N}^{\prime}$, can be obtained.

From the analysis above, it can be observed that the wavelet-transform-based fault location method is described in frequency domain from Step 3 to Step 6. The method is implemented again from Step 7 to Step 9, and the traveling wave is corrected in frequency domain.

\section{Simulations and Results Analysis}

5.1. The Model of Simulation. A model for $500 \mathrm{kV}$ transposed transmission line is constructed in ATP/EMTP, as shown in Figure 6(a). The geometry structure of transmission line is shown in Figure 6(b). The classical J. Marti model is adopted for analyzing the frequency characteristic of transmission lines. The voltage measuring terminal is at each transformer and is used to acquire the fault voltage data. The total length of the transmission line is $414 \mathrm{~km}$. The fault distance is $100 \mathrm{~km}$. Besides, an A-phase grounding fault happens.

In ATP/EMTP software, the sample rate is set as $1 \mathrm{MHz}$. The traveling-wave data generated in ATP software is imported into MATLAB software. In MATLAB, the correction method is implemented. In this paper, $\alpha$-mode component is used for fault location. Due to the fact that the velocity is dependent on frequency, the $\alpha$-mode velocity of traveling wave is calculated at $50 \mathrm{~Hz}$, which is $2.9724 \times$ $10^{5} \mathrm{~km} / \mathrm{s}$. 


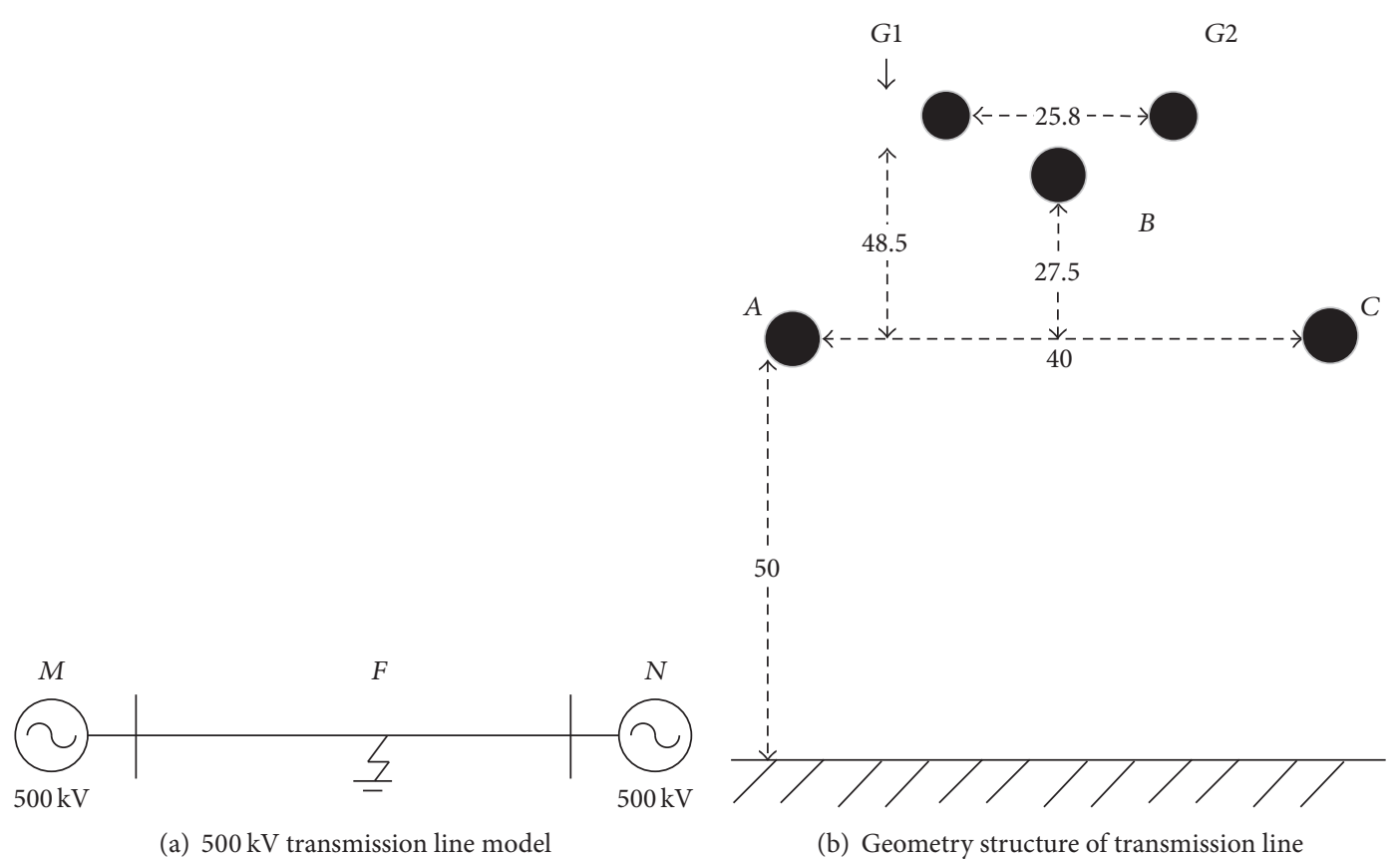

FIGURE 6: Model of transmission line.

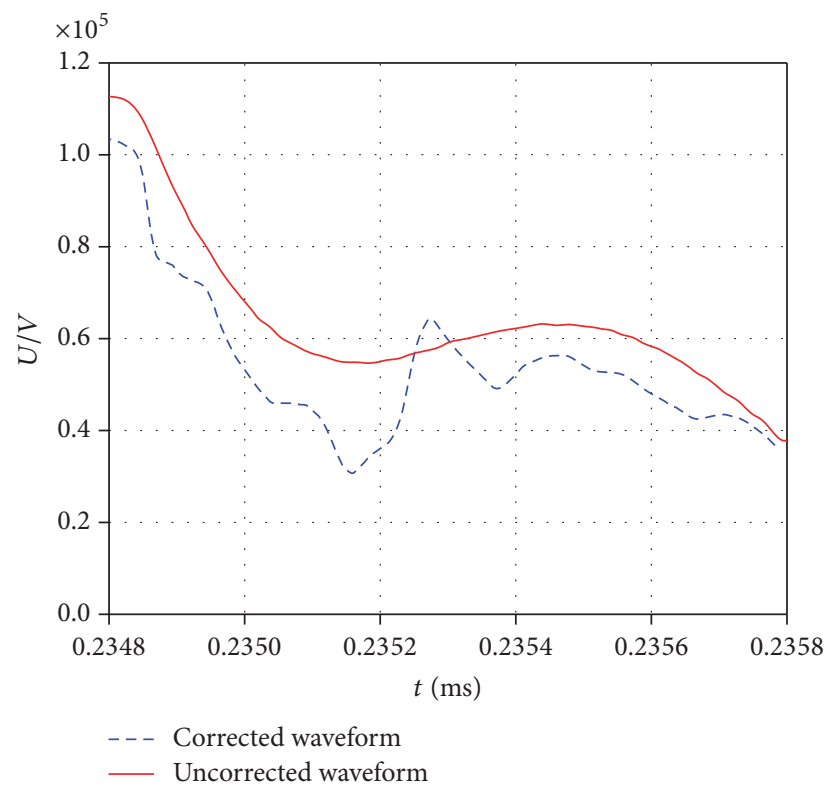

FIGURE 7: Corrected waveforms with the distance of $345 \mathrm{~km}$.

5.2. Simulation Waveform Analysis. When we select several discrete frequencies and calculate the propagation function of each frequency with $R, L$, and $C$ parameters by using Carson formulation, the propagation function $A(\omega)$ can be obtained by using (19).

When we obtain $A(\omega)$, it is used for correcting the receiving traveling wave by using (21). The corrected traveling wave is shown in Figure 7. At the moment, the transmission line is grounded in A phase, the transition resistance is $10 \Omega$, and the fault inception angle is $106^{\circ}$.

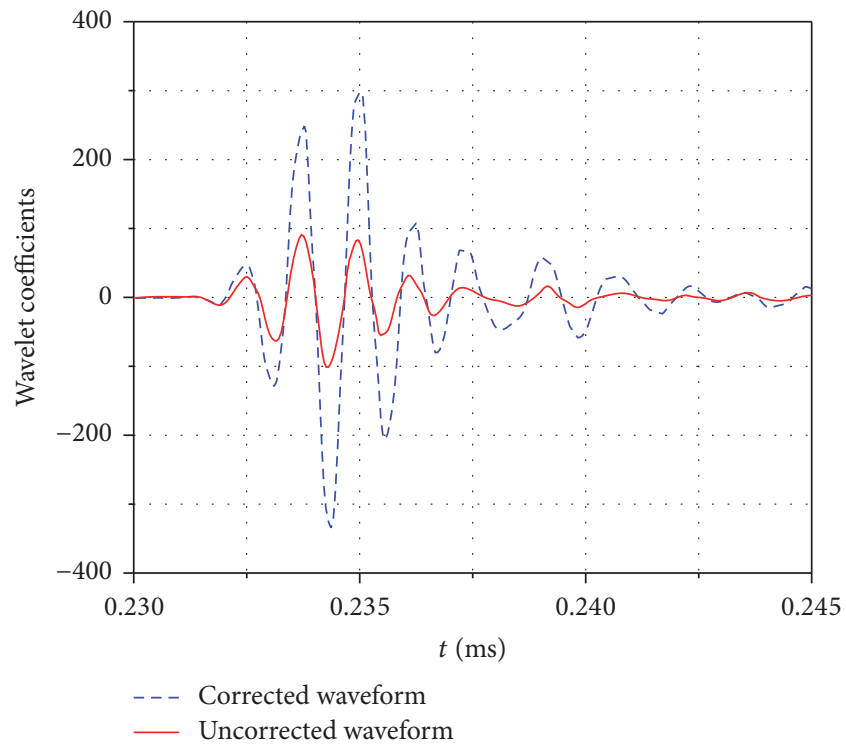

FIGURE 8: Comparisons of the wavelet coefficients.

As can be seen from Figure 7, the fall time of the corrected traveling wave becomes shorter than of the uncorrected one, which enhances the singularity of the traveling wave. That is to say, the corrected traveling wave shows more singularity than the uncorrected one. The correction method proposed in this paper makes the wavefront easier to be detected by the extraction algorithms, such as wavelet transform and HHT. In this paper, the comparison analysis is also implemented in wavelet field. As shown in Figure 8, the modulus maximum of uncorrected wavelet component is less than that of corrected wavelet component. 
TABLE 1: Error under different transition resistance values (transposed circuit).

\begin{tabular}{|c|c|c|c|c|}
\hline \multirow{2}{*}{ Transition resistance } & \multicolumn{2}{|c|}{ The traditional method } & \multicolumn{2}{|c|}{ The proposed method } \\
\hline & Location results & Error & Location results & Error \\
\hline 10 & 100.3 & 0.10 & 100.23 & 0.07 \\
\hline 50 & 99.50 & 0.18 & 99.60 & 0.13 \\
\hline 100 & 99.34 & 0.22 & 99.62 & 0.12 \\
\hline 500 & 98.70 & 0.43 & 99.30 & 0.23 \\
\hline
\end{tabular}

TABLE 2: Error under different transition resistance values (untransposed circuit).

\begin{tabular}{lccc}
\hline Transition resistance & $\begin{array}{c}\text { The traditional method } \\
\text { Location results }\end{array}$ & \multicolumn{2}{c}{$\begin{array}{c}\text { The proposed method } \\
\text { Location results }\end{array}$} \\
\hline 10 & 100.57 & 0.19 & 100.30 \\
50 & 101.20 & 0.40 & 100.56 \\
100 & 98.75 & 0.42 & 99.18 \\
500 & 101.02 & 0.34 & 0.19 \\
\hline
\end{tabular}

TABLE 3: Error under different inception angles (transposed circuit).

\begin{tabular}{|c|c|c|c|c|}
\hline \multirow{2}{*}{ Fault inception angle } & \multicolumn{2}{|c|}{ The traditional method } & \multicolumn{2}{|c|}{ The proposed method } \\
\hline & Location results & Error & Location results & Error \\
\hline $18^{\circ}$ & 100.60 & 0.20 & 100.42 & 0.07 \\
\hline $36^{\circ}$ & 100.31 & 0.10 & 100.22 & 0.10 \\
\hline $72^{\circ}$ & 100.86 & 0.29 & 99.96 & 0.18 \\
\hline $90^{\circ}$ & 100.80 & 0.27 & 100.78 & 0.26 \\
\hline
\end{tabular}

5.3. Results of Fault Location. In this paper, we compare the fault location accuracy between the traditional method and the proposed method. The traditional method is based on the wavelet transform (Morlet wavelet function) and it is not corrected by the correction method proposed in this paper. In order to test the applicability of the proposed method, various fault conditions are simulated, respectively. In the simulated experiments, the error of fault location is calculated as a performance index by the following equation:

$$
\text { error }=\frac{\left|l^{\prime}-l\right|}{S} \times 100 \% \text {, }
$$

where $l^{\prime}$ is the calculated fault distance, $l$ is the actual fault distance, and $S$ is the length of total transmission line.

\subsubsection{Performance of Different Transition Resistance Values.} The transition resistance directly affects the voltage amplitude of the initial traveling wave. In this paper, we set the fault inception angle to analyze the errors of fault location, when the transition resistance varies from $10 \Omega$ to $500 \Omega$. The distance between the fault point and the measuring terminal is $100 \mathrm{~km}$, the fault type is A-G, and the fault inception angle is $106^{\circ}$. The simulated results are shown in Tables 1 and 2 . As can be seen from the table, the location error of the proposed method becomes smaller than that of the traditional one, no matter what the transition resistance is.

5.3.2. Performance of Different Inception Angles. The fault inception angle is one of the most effective parameters which influence the accuracy of the traveling-wave-based fault location. In order to test the influence of fault inception angle, the simulated cases are constructed with the fault inception angle varied from $18^{\circ}$ to $90^{\circ}$. At the moment, the fault type is A-G, the transition resistance is $10 \Omega$, and the fault distance is 100 kilometers. The experiment results are shown in Tables 3 and 4 . The proposed method also shows great adaptability to the different inception angles.

5.3.3. Performance of Different Fault Types. To investigate the effect of different fault types based on the proposed method in this paper, several experiments have been implemented and experiment results are shown in Tables 5 and 6 . In these experiments, all transition resistances are $10 \Omega$, the fault inception angle is $106^{\circ}$, and the fault distance is 100 kilometers.

5.3.4. Performance of Antinoise. To investigate the performance of antinoise under different levels, several experiments have been implemented. In these experiments, the transition resistance is $10 \Omega$ with BC-G fault, the fault inception angle is $106^{\circ}$, and the fault distance is 100 kilometers. Since noise has no meaningful information about fault location, it would be rational to define a threshold to reject the noise-associated WT coefficient [8]. Simulation results in Table 7 reveal that the additional noise has little influence on traveling-wavebased fault location.

5.3.5. Performance Analysis of Geometry Parameters. The production error of the towers supporting transmission line 
TABLE 4: Error under different inception angles (untransposed circuit).

\begin{tabular}{|c|c|c|c|c|}
\hline \multirow{2}{*}{ Fault inception angle } & \multicolumn{2}{|c|}{ The traditional method } & \multicolumn{2}{|c|}{ The proposed method } \\
\hline & Location results & Error & Location results & Error \\
\hline $18^{\circ}$ & 100.85 & 0.28 & 100.50 & 0.17 \\
\hline $36^{\circ}$ & 100.62 & 0.21 & 100.40 & 0.13 \\
\hline $72^{\circ}$ & 99.33 & 0.22 & 99.60 & 0.13 \\
\hline $90^{\circ}$ & 100.56 & 0.19 & 100.33 & 0.11 \\
\hline
\end{tabular}

TABLE 5: Error under different fault types (transposed circuit).

\begin{tabular}{|c|c|c|c|c|}
\hline \multirow{2}{*}{ Fault type } & \multicolumn{2}{|c|}{ The traditional method } & \multicolumn{2}{|c|}{ The proposed method } \\
\hline & Location results & Error & Location results & Error \\
\hline$A-G$ & 100.30 & 0.10 & 100.23 & 0.08 \\
\hline$B-G$ & 99.50 & 0.17 & 99.63 & 0.12 \\
\hline $\mathrm{BC}$ & 99.21 & 0.26 & 100.45 & 0.15 \\
\hline BC-G & 100.87 & 0.29 & 100.14 & 0.05 \\
\hline $\mathrm{ABC}-\mathrm{G}$ & 99.25 & 0.25 & 99.45 & 0.18 \\
\hline
\end{tabular}

TABLE 6: Error under different fault types (untransposed circuit).

\begin{tabular}{|c|c|c|c|c|}
\hline \multirow{2}{*}{ Fault type } & \multicolumn{2}{|c|}{ The traditional method } & \multicolumn{2}{|c|}{ The proposed method } \\
\hline & Location results & Error & Location results & Error \\
\hline A-G & 100.57 & 0.19 & 100.30 & 0.10 \\
\hline$B-G$ & 99.54 & 0.15 & 99.64 & 0.12 \\
\hline $\mathrm{BC}$ & 101.05 & 0.35 & 100.85 & 0.28 \\
\hline BC-G & 100.78 & 0.26 & 100.23 & 0.08 \\
\hline ABC-G & 100.50 & 0.16 & 100.26 & 0.09 \\
\hline
\end{tabular}

TABLE 7: Error under different noise levels (transposed circuit).

\begin{tabular}{|c|c|c|c|c|}
\hline \multirow{2}{*}{ Noise (unit: dBw) } & \multicolumn{2}{|c|}{ The traditional method } & \multicolumn{2}{|c|}{ The proposed method } \\
\hline & Location results & Error & Location results & Error \\
\hline 10 & 100.78 & 0.26 & 100.14 & 0.05 \\
\hline 20 & 100.78 & 0.26 & 100.15 & 0.05 \\
\hline 30 & 99.21 & 0.2633 & 100.30 & 0.10 \\
\hline 40 & 100.76 & 0.2533 & 100.21 & 0.07 \\
\hline
\end{tabular}

is very low, and it is within $1 \mathrm{~cm}$. The geometrical parameters of the corner tower and the tower in substation are sometimes different from other towers along the transmission line. The number of the corner towers and the towers in substation is very small and they can be omitted. Therefore, from the point of view of theoretical calculation, we assume that the relative position between line conductors is unchanged. On the other hand, the height of line conductors will sometimes increase when the transmission line passes through a road, village, city, mountain, and so forth. The change is very complicated; it is very difficult to model the real transmission line. In the paper, for analyzing the applicability of the proposed method, the length of the transmission line which is supported by the changed tower is $20 \mathrm{~km}$. In these experiments, the transition resistance is $10 \Omega$ with $\mathrm{A}-\mathrm{G}$ fault, the fault inception angle is $106^{\circ}$, and the fault distance is 100 kilometers. Simulation results in Table 8 reveal that the proposed method can also improve the accuracy of fault location when the height of tower varies.

\section{Conclusions}

In this paper, the dispersion characteristic of traveling wave is analyzed in time and frequency domain, respectively. When traveling wave travels along transmission line, the fall or rise time of the wavefront will become long with the increase of propagation distance. Thus, the singularity of the transient wavefront decreases. In this paper, a novel doubleended fault location method has been proposed to overcome the dispersion effect of traveling wave. In the method, a correction algorithm for overcoming the dispersion effect of traveling wave enhances the singularity of the transient traveling wave. The proposed method is tested under various experiment conditions, such as different fault distances, 
TABLE 8: Error under different tower heights (transposed circuit).

\begin{tabular}{|c|c|c|c|c|}
\hline \multirow{2}{*}{ Tower height (mile) } & \multicolumn{2}{|c|}{ The traditional method } & \multicolumn{2}{|c|}{ The proposed method } \\
\hline & Location results & Error & Location results & Error \\
\hline 0 & 100.60 & 0.20 & 100.14 & 0.05 \\
\hline+5 & 100.47 & 0.16 & 100.22 & 0.07 \\
\hline+10 & 99.18 & 0.27 & 100.45 & 0.15 \\
\hline+20 & 100.80 & 0.27 & 100.55 & 0.18 \\
\hline
\end{tabular}

different transition resistances, and different fault inception angles. The simulation experiments demonstrate that the proposed method is better than the traditional travelingwave-based fault location method. Furthermore, the novel method is suitable for both transposed and untransposed transmission lines. All of the advantages prove that the proposed method is available. However, there are several other parameters which affect the slope of traveling wave, such as the impedance characteristic of traveling-wave measurement equipment and shunt reactor connected to the line. Future work will consider those parameters.

\section{Competing Interests}

The author declares that there are no competing interests regarding the publication of this paper.

\section{Acknowledgments}

This work was supported by the Fundamental Research Funds for the Central Universities (no. 13MS68) and Hebei Province Natural Science Foundation of China, Youth Science Fund (no. E2013502267).

\section{References}

[1] T. W. Stringfield, D. J. Marihart, and R. F. Stevens, "Fault location methods for overhead lines," Transactions of the American Institute of Electrical Engineers. Part III: Power Apparatus and Systems, vol. 76, no. 3, pp. 518-529, 1957.

[2] M. M. Saha, J. Izykowski, and E. Rosolowski, Fault Location on Power Networks, Power Systems, Springer, London, UK, 2010.

[3] P. Gale and R. Burnett, "A study of power line response to lightning using GPS based travelling wave fault locators and the US national lightning detection network," in Proceedings of the Fault and Disturbance Analysis and Precise Measurements Power Systems Conference, Arlington, Va, USA, 1996.

[4] B. J. Cory and P. F. Gale, "Satellites for power system applications," Power Engineering Journal, vol. 7, no. 5, pp. 201-207, 1993.

[5] F. H. Magnago and A. Abur, "Fault location using wavelets," IEEE Transactions on Power Delivery, vol. 13, no. 4, pp. 14751480, 1998.

[6] C. Y. Evrenosoglu and A. Abur, "Travelling wave based fault location for teed circuits," IEEE Transactions on Power Delivery, vol. 20, no. 2, pp. 1115-1121, 2005.

[7] D. Spoor and J. G. Zhu, "Improved single-ended traveling-wave fault-location algorithm based on experience with conventional substation transducers," IEEE Transactions on Power Delivery, vol. 21, no. 3, pp. 1714-1720, 2006.
[8] P. Jafarian and M. Sanaye-Pasand, "A traveling-wave-based protection technique using wavelet/PCA analysis," IEEE Transactions on Power Delivery, vol. 25, no. 2, pp. 588-599, 2010.

[9] W. Zhao, Y. H. Song, and W. R. Chen, "Improved GPS travelling wave fault locator for power cables by using wavelet analysis," International Journal of Electrical Power and Energy Systems, vol. 23, no. 5, pp. 403-411, 2001.

[10] S. Lin, Z. Y. He, X. P. Li, and Q. Q. Qian, “Travelling wave timefrequency characteristic-based fault location method for transmission lines," IET Generation, Transmission and Distribution, vol. 6, no. 8, pp. 764-772, 2012.

[11] A. Borghetti, M. Bosetti, M. Di Silvestro, C. A. Nucci, and M. Paolone, "Continuous-wavelet transform for fault location in distribution power networks: definition of mother wavelets inferred from fault originated transients," IEEE Transactions on Power Systems, vol. 23, no. 2, pp. 380-388, 2008.

[12] A. Borghetti, M. Bosetti, C. A. Nucci, M. Paolone, and A. Abur, "Integrated use of time-frequency wavelet decompositions for fault location in distribution networks: theory and experimental validation," IEEE Transactions on Power Delivery, vol. 25, no. 4, pp. 3139-3146, 2010.

[13] F. Zhang, J. Liang, L. Zhang, and Z. Yun, "Novel method about traveling wave fault location based on treble terminal measurement data for transmission lines," Dianli Xitong Zidonghua/Automation of Electric Power Systems, vol. 32, no. 8, pp. 69-72, 2008.

[14] Z. Wu, Z. Li, X. Qin et al., "A novel double terminal traveling wave fault location method not influenced by wave speed," in Proceedings of the IEEE Transmission and Distribution Conference and Exposition: Asia and Pacific, September 2009.

[15] F. V. Lopes, K. M. Silva, F. B. Costa, W. L. A. Neves, and D. Fernandes, "Real-time traveling-wave-based fault location using two-terminal unsynchronized data," IEEE Transactions on Power Delivery, vol. 30, no. 3, pp. 1067-1076, 2015.

[16] X. Zeng, Research on the new principle of fault detection and location of power line and the realization of information fusion [Ph.D. thesis], Huazhong University of Science and Technology, 2000.

[17] $\mathrm{X}$. Hu, The on-line measurement of the traveling wave velocity in fault location system [Ph.D. thesis], Sichuan University, 2003.

[18] J. Qin, X. Chen, and J. Zheng, "Study on dispersion of travelling wave in transmission line," Proceedings of the Chinese Society of Electrical Engineering, vol. 19, no. 9, pp. 27-35, 1999.

[19] J. Qin, Z. Huang, H. Yang, and Y.-F. Qiu, "Study on traveling wave propagation in double-circuit parallel transmission line," Proceedings of the Chinese Society of Electrical Engineering, vol. 24, no. 5, pp. 30-34, 2004.

[20] J. Qin, W. Ge, J. Qiu et al., "Analysis on main influencing factors for transmission lines fault location precision based on traveling wave," Power System Technology, vol. 31, no. 2, pp. 27-35, 2007. 
[21] J. R. Marti, H. W. Dommel, L. Marti et al., "Approximate transformation matrices for unbalanced transmission system," in Proceedings of the 9th Power Systems Computation Conference (PSCC '87), vol. 8, pp. 416-422, 1987.

[22] J. A. Brandão Faria and J. Hildemaro Briceño, "On the modal analysis of asymmetrical three-phase transmission lines using standard transformation matrices," IEEE Transactions on Power Delivery, vol. 12, no. 4, pp. 1760-1765, 1997.

[23] J. R. Marti, L. Marti, and H. W. Dommel, "Transmission line models for steady-state and transients analysis," in Proceedings of the Joint International Power Conference Athens Power Tech (APT '93), vol. 2, pp. 744-750, Athens, Greece, September 1993.

[24] S. Mallat, A wavelet tour of signal processing, Elsevier/Academic Press, Amsterdam, Netherlands, 3rd edition, 2009.

[25] S. Mallat and W. L. Hwang, "Singularity detection and processing with wavelets," IEEE Transactions on Information Theory, vol. 38, no. 2, pp. 617-643, 1992.

[26] E. S. M. Mok and G. I. Costache, "Skin-effect considerations on transient response of a transmission line excited by aa electromagnetic pulse," IEEE Transactions on Electromagnetic Compatibility, vol. 34, no. 3, pp. 320-329, 1992. 


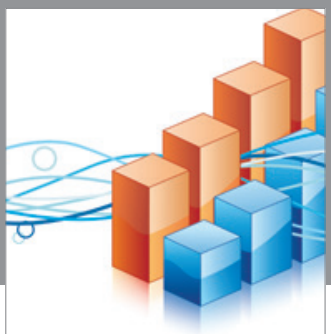

Advances in

Operations Research

vatem alat4

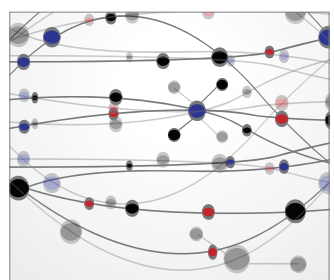

\section{The Scientific} World Journal
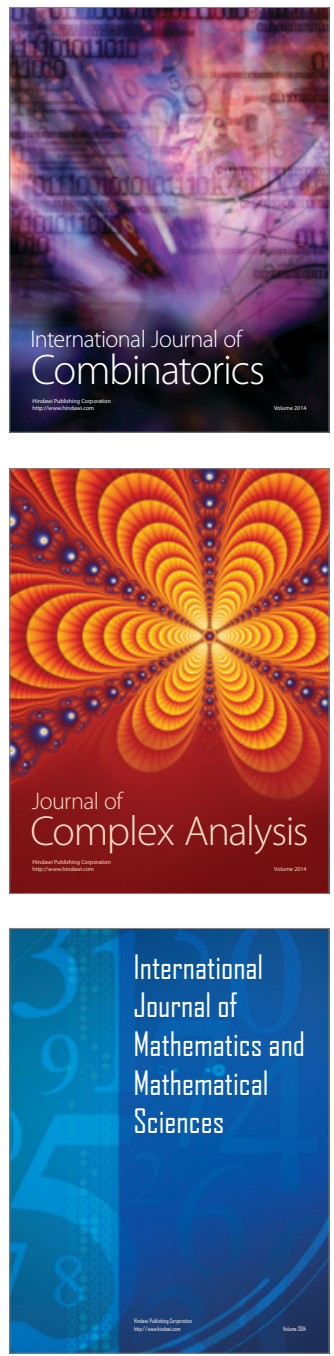
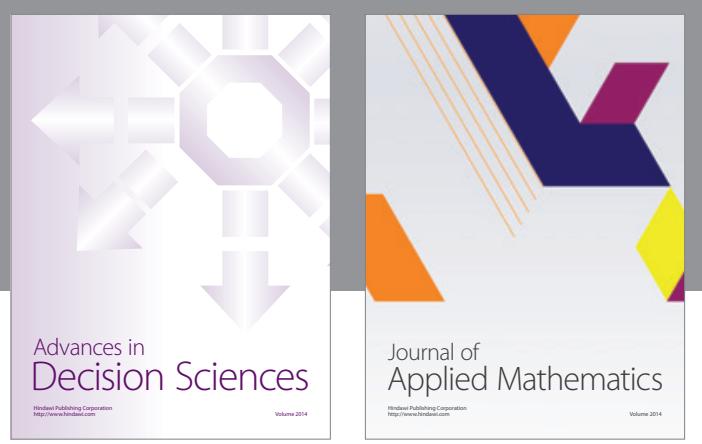

Algebra

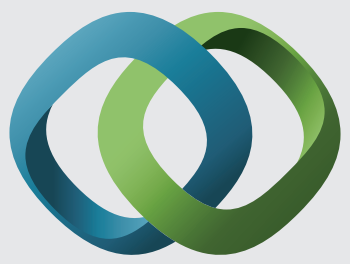

\section{Hindawi}

Submit your manuscripts at

https://www.hindawi.com
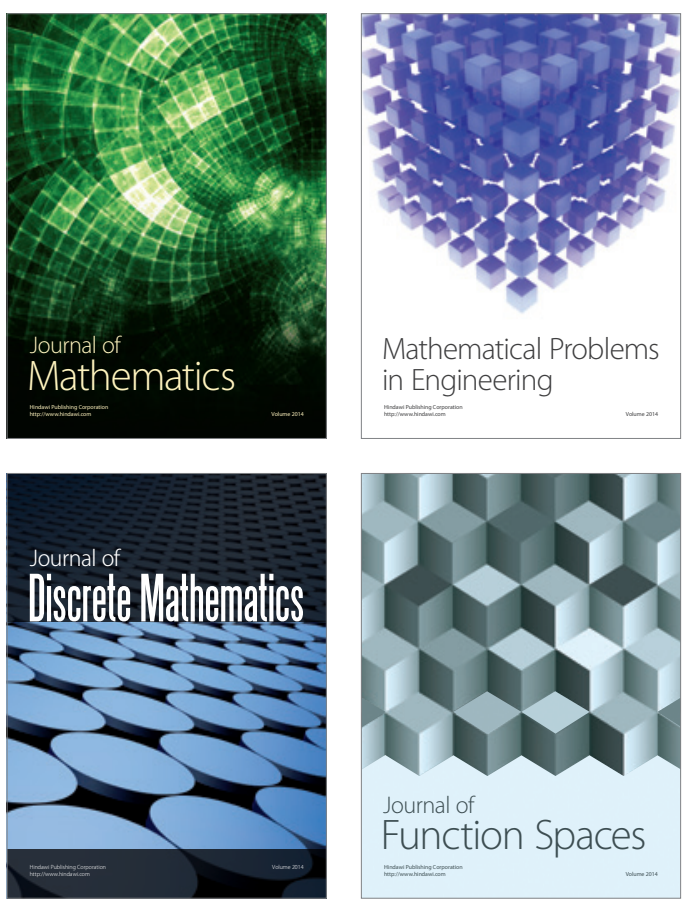

Mathematical Problems in Engineering
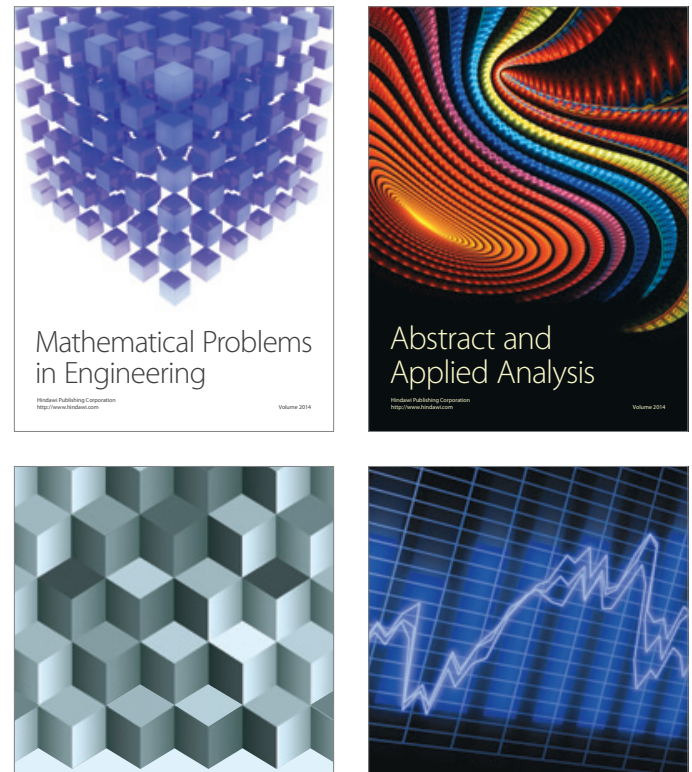

Journal of

Function Spaces

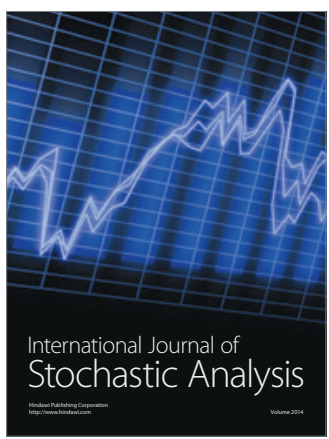

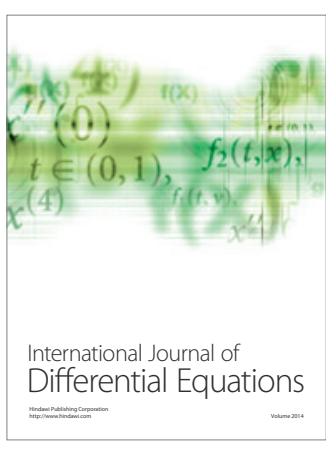
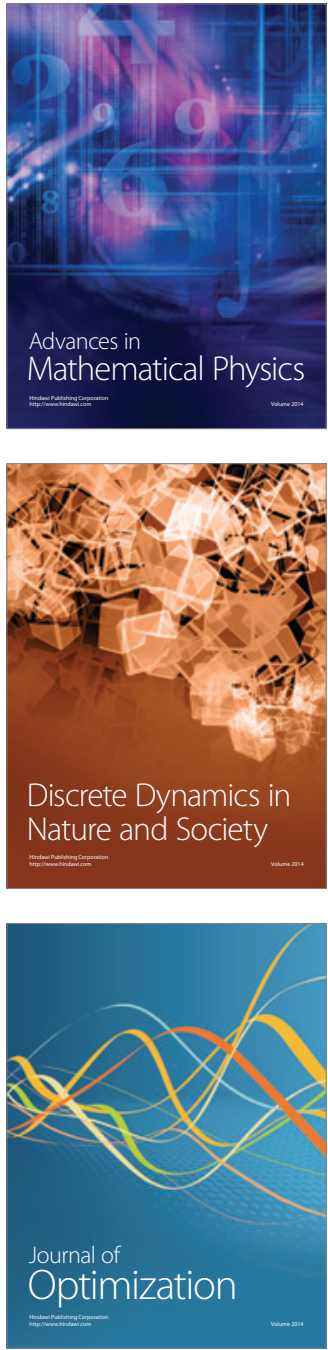